\title{
DEFINABLE GROUP EXTENSIONS IN SEMI-BOUNDED O-MINIMAL STRUCTURES
}

\author{
MÁRIO J. EDMUNDO AND PANTELIS E. ELEFTHERIOU
}

\begin{abstract}
In this note we show: Let $\mathcal{R}=\langle R,<,+, 0, \ldots\rangle$ be a semi-bounded (respectively, linear) o-minimal expansion of an ordered group, and $G$ a group definable in $\mathcal{R}$ of linear dimension $m$ ([Ed1]). Then $G$ is a definable extension of a bounded (respectively, definably compact) definable group $B$ by $\left\langle R^{m},+\right\rangle$.
\end{abstract}

\section{INTRODUCTION}

We fix throughout this paper an o-minimal expansion $\mathcal{R}=\langle R,<,+, 0, \ldots\rangle$ of an ordered group. By "definable" we mean "definable in $\mathcal{R}$ " possibly with parameters. A group $G=\langle G, \cdot\rangle$ is said to be definable if both its domain and its group operation are definable.

The study of groups definable in o-minimal structures was originated in [Pi1], where it was shown that every such group admits a unique definable manifold topology that makes it into a topological group, called $t$-topology. Ever since numerous results have been established manifesting the close connection of these groups with real Lie groups. The climax of these results was Pillay's Conjecture for definably compact groups from [Pi2] and its solution in case $\mathcal{R}$ is linear ([ElSt]) and in case $\mathcal{R}$ expands a real closed field ([HPP]). More recently, the conjecture was solved in the intermediate case where $\mathcal{R}$ is semi-bounded ([Pet2]), by a reduction of the conjecture to the field case. This concluded the proof of Pillay's Conjecture in arbitrary o-minimal expansions of ordered groups.

In this note we suggest the possibility of describing the fine structure of groups definable in a semi-bounded $\mathcal{R}$. We prove (Theorem 1.5) that every such group is a definable extension of a definable bounded group by $\left\langle R^{m},+\right\rangle$, where $m$ is the linear dimension of $G$, introduced in [Ed1]. If $\mathcal{R}$ is linear, then the bounded group is actually definably compact.

Let us now introduce the terminology of this paper. For general background on o-minimal structures, we refer the reader to $[\mathrm{vdD}]$, and for a recent survey on groups definable in o-minimal structures, to $[\mathrm{Ot}]$.

Definition 1.1. Let $G, H$ and $K$ be definable groups. We say that $G$ is a definable extension of $K$ by $H$ if there is a definable surjective homomorphism $\pi: G \longrightarrow K$ whose kernel is definably isomorphic to $H$; that is to say, if there is a short exact sequence

$$
0 \longrightarrow H \longrightarrow G \stackrel{\pi}{\longrightarrow} K \longrightarrow 0,
$$

Date: August 6, 2008 - Revised: December 1, 2008.

2000 Mathematics Subject Classification. 03C64, $20 \mathrm{~K} 35$.

Key words and phrases. O-minimal structures, Group extensions.

The first author was partially supported by FEDER and FCT-Plurianual 2007.

The second author was supported by the FCT grant SFRH/BPD/35000/2007. 
where all maps involved are definable homomorphisms.

By definable choice, if $G$ is a definable extension of $K$ by $H$, then $G$ is in definable bijection with $H \times K$ as abstract sets, but not necessarily definably isomorphic to the direct product of $H$ and $K$.

Definition 1.2. Let $\Lambda_{p . e}$ be the division ring of all partial definable endomorphisms of $\langle R,<,+, 0\rangle$, and $\mathcal{B}$ the collection of all bounded definable sets. Then $\mathcal{R}$ is called linear ([LP]) if every definable set is already definable in $\left\langle R,<,+, 0,\{\lambda\}_{\lambda \in \Lambda_{p . e}}\right\rangle$, and it is called semi-bounded ([Ed1, Pet1]) if every definable set is already definable in $\left\langle R,<,+, 0,\{\lambda\}_{\lambda \in \Lambda_{p . e}},\{B\}_{B \in \mathcal{B}}\right\rangle$.

Obviously, if $\mathcal{R}$ is linear then it is semi-bounded.

Let $\Lambda$ be the division ring of all definable endomorphisms of $\langle R,<,+, 0\rangle$, which is a subring of $\Lambda_{p . e}$. Clearly, $\mathcal{R}$ is semi-bounded if and only if every definable set is already definable in $\left\langle R,<,+, 0,\{\lambda\}_{\lambda \in \Lambda},\{B\}_{B \in \mathcal{B}}\right\rangle$. We fix this $\Lambda$.

For $\lambda=\left(\lambda_{1}, \ldots, \lambda_{n}\right) \in \Lambda^{n}$ and $x \in R$, we denote $\lambda x=\left(\lambda_{1} x, \ldots, \lambda_{n} x\right)$.

Definition 1.3. Let $k \in \mathbb{N}$. A $k$-cone $C \subseteq R^{n}$ is a definable set of the form

$$
\left\{b+\sum_{i=1}^{k} v_{i} t_{i}: b \in B, t_{1}, \ldots, t_{k} \in R^{>0}\right\},
$$

where $B \subseteq R^{n}$ is a bounded definable set and $v_{1}, \ldots, v_{k} \in \Lambda^{n}$ are linearly independent (that is, $\forall t_{1}, \ldots, t_{k} \in R, \sum_{i=1}^{k} v_{i} t_{i}=0$ if and only if $t_{1}=\cdots=t_{k}=0$ ). So a 0 cone is just a bounded definable set. A cone is a $k$-cone, for some $k \in \mathbb{N}$. We say that the cone $C$ is normalized if for each $x \in C$ there are unique $b \in B, t_{1}, \ldots, t_{k} \in R^{>0}$ such that $x=b+\sum_{i=1}^{k} v_{i} t_{i}$. In this case, we write:

$$
C=B+\sum_{i=1}^{k} v_{i} t_{i}
$$

Definition 1.4. Let $X \subseteq R^{n}$ be a definable set. The linear dimension of $X$, denoted by $\lim (X)$, is defined to be

$$
\lim (X)=\max \{k: X \text { contains a } k \text {-cone }\} \text {. }
$$

In what follows, if $G$ is a definable group, then by a definable path $\varphi:(p, q) \subseteq$ $M \rightarrow G$ we mean a definable map which is continuous with respect to the $t$-topology in the range. Recall from $[\mathrm{PeS}]$ that a definable group $G$ is called definably compact if for every definable path $\varphi:(p, q) \subseteq M \rightarrow G,-\infty \leq p<q \leq \infty$, there is $z \in G$ such that $\lim _{t \rightarrow q^{-}}^{G} \sigma(t)=z$, where the upper index " $G$ " indicates, again, that the limit is taken with respect to the $t$-topology in the range.

We note that in a semi-bounded structure, the image of a bounded definable set under a definable bijection is also bounded (see Fact 2.1 below).

Theorem 1.5. Assume that $\mathcal{R}$ is a semi-bounded (respectively, linear) o-minimal expansion of an ordered group, and $G$ a definable group with $\operatorname{ldim}(G)=m$. Then $G$ is a definable extension of a bounded (respectively, definably compact) definable group $B$ by $\left\langle R^{m},+\right\rangle$ :

$$
0 \longrightarrow\left\langle R^{m},+\right\rangle \longrightarrow G \longrightarrow B \longrightarrow 0
$$


In Section 2, we prove Theorem 1.5 for the semi-bounded case. The proof is split into two parts. In the first part we prove that a definable group $K$ of dimension one and linear dimension one is definably isomorphic to $\langle R,+\rangle$. The argument uses the Structure Theorem for definable sets from [Ed1], the definable order on $K$ extracted from [Raz], and a result from [MS]. In the second part, we use the analysis of solvable definable groups from [Ed2] in order to decompose $G$ into a bounded definable group and definable subgroups of dimension one and linear dimension one.

In Section 3, we prove that if $\mathcal{R}$ is linear, then a definable group is definably compact if and only if it is bounded. The linear version of Theorem 1.5 follows.

In the rest of this Introduction, we prove some basic facts for one-dimensional groups, making heavy use of the analysis in [Raz].

Fact 1.6. Let $K=\left\langle K, *, 0_{K}\right\rangle$ be a definably connected definable group of dimension one which is not definably compact. Then there is a definable total order $<_{K}$ on $K$, such that $\left\langle K,<_{K}, *, 0_{K}\right\rangle$ is an o-minimal ordered group. Moreover,

$$
\begin{aligned}
& \text { every definable function } f: X \subseteq\langle R,<\rangle \longrightarrow\left\langle K,<_{K}\right\rangle \text { is piecewise } \\
& \text { either constant, or strictly }<_{K} \text {-monotonous and continuous. }
\end{aligned}
$$

and the Intermediate Value Theorem (IVT) holds for definable continuous functions of the form $f: X \subseteq\langle R,<\rangle \longrightarrow\left\langle K,<_{K}\right\rangle$.

Proof. By [Raz, Proposition 2], $K \backslash\{$ point $\}$ has either one or two definably connected components. Since $K$ is not definably compact, the latter case holds. By [Raz, Proposition 2] and its proof, there is a definable total order $<_{K}$ on $K$, such that

$$
\begin{aligned}
& \text { for every chart map } f: I \longrightarrow K \text { from the } t \text {-topology of } K, \\
& \text { where } I \subseteq R \text { is an open interval, } a<b \leftrightarrow f(a)<_{K} f(b) .
\end{aligned}
$$

But by definition of the $t$-topology, (2) implies that the $t$-topology and the order $<_{K}$-topology coincide (locally, and thus everywhere). Moreover, (2) easily implies that $\left\langle K,<_{K}, *, 0_{K}\right\rangle$ is an o-minimal structure and Properties (1) and (IVT) hold. We show that $\left\langle K,<_{K}, *, 0_{K}\right\rangle$ is actually an ordered group. Without loss of generality, we only show invariance from the left (in fact, $K$ is abelian):

$$
\forall x, y, z \in K, y<_{K} z \rightarrow x * y<_{K} x * z .
$$

Fix $y, z \in K$ with $y<_{K} z$, and let $\Gamma=\left\{x \in K: x * y<_{K} x * z\right\}$. Clearly, $\Gamma \neq \emptyset$. We show that $\Gamma$ is clopen in $K$. To see that $\Gamma$ is open in $K$, let $x_{0} \in \Gamma$. If $x_{0}$ is not contained in any open $<_{K}$-interval, then, by o-minimality of $\left\langle K,<_{K}, *, 0_{K}\right\rangle$, either

- for all $x \in K$ close to $x_{0}$ from above, $x * y>_{K} x * z$, or

- for all $x \in K$ close to $x_{0}$ from below $x * y>_{K} x * z$.

In either case

$$
x_{0} * y=\lim _{x \rightarrow x_{0}}^{K} x * y \geq_{K} \lim _{x \rightarrow x_{0}}^{K} x * z=x_{0} * z,
$$

a contradiction. Thus $\Gamma$ is open in $K$. Similarly, $\Gamma$ is closed in $K$.

So now let $K=\left\langle K, *, 0_{K}\right\rangle$ be a definably connected definable group of dimension one which is not definably compact. As an o-minimal ordered group, $K=\left\langle K,<_{K}\right.$ , $\left.*, 0_{K}\right\rangle$ is divisible, torsion-free, and abelian. We denote by $-_{*}$ the inverse map of $K$. We next prove some basic properties to be used in the sequel. 
Since $K$ is not definably compact, there is a definable path $\varphi:(p, q) \longrightarrow K$, $-\infty \leq p<q \leq \infty$, for which there is no $z \in K$ such that $\lim _{t \rightarrow q^{-}}^{K} \varphi(t)=z$. By Property (1), there is some $(r, q), r>p$, on which $\varphi$ is strictly $<_{K}$-monotone and continuous. We may assume that on $(r, q) \varphi$ is strictly $<_{K}$-increasing (otherwise replace $\varphi$ by $\left.-_{*} \varphi(t)\right)$. It is also clearly harmless to assume that $\forall t \in[r, q)$, $\varphi(t)>_{K} \quad 0_{K}$. Let $\infty_{K}=\lim _{t \rightarrow q^{-}} \varphi(t) \in(R \cup\{ \pm \infty\})^{n}$. We have:

$$
\forall z \in K, \exists t_{0} \in(r, q), \forall t \in\left(t_{0}, q\right), \varphi(t)>_{K} z .
$$

Indeed, if the set $A=\left\{z \in K: \forall t_{0} \in(r, q), \exists t \in\left(t_{0}, q\right), \varphi(t)<_{K} z\right\}$ were nonempty, then $\lim _{t \rightarrow q^{-}}^{G} \varphi(t)=\inf _{<_{K}} A$. We then also clearly have: $\forall a>_{K} 0_{K}$,

$$
\forall z \in K, \exists t_{0} \in(r, q), \forall t \in\left(t_{0}, q\right), a * \varphi(t)>_{K} z .
$$

\section{The SEMI-BOUnded CASE}

We begin by recalling some facts.

Fact 2.1 (Fact 1.6 in [Ed1]). The following are equivalent:

(1) $\mathcal{R}$ is semi-bounded.

(2) $\mathcal{R}$ has no poles; that is, there is no definable bijection between a bounded and an unbounded definable set.

(3) [Structure Theorem]: Every definable set $X \subseteq R^{n}$ can be partitioned into finitely many normalized cones. Furthermore, for every definable function $F: X \subseteq R^{n} \longrightarrow R$, there is a partition $\mathcal{C}$ of $X$ into finitely many normalized cones, such that for each $k \in \mathbb{N}$, if $C=B+\sum_{i=1}^{k} v_{i} t_{i}$ is a $k$-cone in $\mathcal{C}$, then there are $\mu_{1}, \ldots, \mu_{k} \in \Lambda$ such that for all $b \in B, t_{1}, \ldots, t_{k} \in R^{>0}$,

$$
F\left(b+\sum_{i=1}^{k} v_{i} t_{i}\right)=F(b)+\sum_{i=1}^{k} \mu_{i} t_{i} .
$$

For the rest of this section, we assume that $\mathcal{R}$ is semi-bounded.

Fact $2.2([\mathrm{Ed} 1])$. Let $X, Y$ be definable sets. Then:

(1) If $f: X \longrightarrow R^{n}$ is a definable injective function, then $\operatorname{ldim}(X)=\operatorname{ldim}(f(X))$.

(2) $X$ is bounded if and only if $\operatorname{ldim}(X)=0$.

(3) $\operatorname{ldim}(X \times Y)=\operatorname{ldim}(X)+\operatorname{ldim}(Y)$.

Proof. We prove (3) which is not explicitly contained in [Ed1]. To see that $\operatorname{ldim}(X \times$ $Y) \geq \operatorname{ldim}(X)+\operatorname{ldim}(Y)$, let $C_{X}=b_{X}+\sum_{i=1}^{\operatorname{ldim}(X)} v_{i} t_{i}$ be a $\operatorname{ldim}(X)$-cone contained in $X$ and $C_{Y}=b_{Y}+\sum_{i=1}^{\operatorname{ldim}(Y)} u_{i} s_{i}$ a $\operatorname{ldim}(Y)$-cone contained in $Y$. Then $C_{X} \times$ $C_{Y}=\left(b_{X}, b_{Y}\right)+\sum_{i=1}^{\operatorname{ldim}(X)}\left(v_{i}, 0\right) t_{i}+\sum_{i=1}^{\operatorname{ldim}(Y)}\left(0, u_{i}\right) s_{i}$ is a $(\operatorname{ldim}(X)+\operatorname{ldim}(Y))$-cone contained in $X \times Y$.

To see that $\operatorname{ldim}(X \times Y) \leq \operatorname{ldim}(X)+\operatorname{ldim}(Y)$, let $C=b+\sum_{i=1}^{k} v_{i} t_{i}$ be a $k$-cone contained in $X \times Y$. Then the projection $\pi_{X}(C)$ onto $X$ is a $k_{X}$-cone, where $k_{X}$ is the maximum number of linearly independent elements among $\pi_{X}\left(v_{i}\right)$. Similarly, we can obtain a $k_{Y}$-cone $\pi_{Y}(C)$ contained in $Y$. But then $\pi_{X}(C) \times \pi_{Y}(C)$ is a $\left(k_{X}+k_{Y}\right)$ cone in $X \times Y$ that contains $C$, and hence $k \leq k_{X}+k_{Y} \leq \operatorname{ldim}(X)+\operatorname{ldim}(Y)$.

Fact 2.3. Every definably compact definable group is bounded.

Proof. See, for example, [ElSt, Lemma 3.7]. An assumption that $\mathcal{R}$ is linear is also existent there, but the proof uses only the fact that $\mathcal{R}$ has no poles. 
Now let $K=\left\langle K, *, 0_{K}\right\rangle$ be a definable group with $\operatorname{dim}(K)=\operatorname{ldim}(K)=1$. Then $K$ contains an 1-cone $C^{\prime}$ of the form $C=b^{\prime}+\lambda^{\prime} t, \lambda^{\prime} \neq 0$. On the other hand, by Facts $2.2(2)$ and $2.3, K$ is not definably compact. So let $\varphi:(p, q) \longrightarrow K$, $-\infty \leq p<q \leq \infty$, be a definable path as in the Introduction, and recall, by definition, $\lim _{t \rightarrow q^{-}} \varphi(t)=\infty_{K}$. We are next going to show that there is actually a normalized 1-cone $C=b+\lambda t$ contained in $K$ such that $\lim _{t \rightarrow \infty}(b+\lambda t)=\infty_{K}$.

By Property (1) from the Introduction, there is some $\left(r^{\prime}, \infty\right), r^{\prime}>0$, on which the map $\sigma: t \longmapsto b^{\prime}+\lambda^{\prime} t$ is strictly $<_{K}$-monotone and continuous. We may assume that on $\left(r^{\prime}, \infty\right) \sigma$ is strictly $<_{K}$-increasing (otherwise, replace $<_{K}$ by $>_{K}$ ).

Claim 2.4. The image $\varphi((r, q))$ is unbounded.

Proof. Assume, towards a contradiction, that $\varphi((r, q))$ is bounded. Let $C=b^{\prime}+\lambda^{\prime} t$ be as above. By Property (3) from the Introduction, we can pick an element $r_{0} \in(r, q)$ such that $\varphi\left(r_{0}\right)>_{K} \sigma\left(r^{\prime}\right)$. Let $c:=\varphi\left(r_{0}\right)-_{*} \sigma\left(r^{\prime}\right)>_{K} 0_{K}$. Then the map

$$
f:\left(r^{\prime}, \infty\right) \longrightarrow K, \text { with } t \longmapsto \sigma(t) * c,
$$

is a strictly $<_{K}$-increasing map with image inside $\varphi\left(\left(r_{0}, q\right)\right)$, contradicting the fact that $\mathcal{R}$ has no poles.

This implies that $q=\infty$, since, otherwise, $\varphi_{\uparrow(r, q)}$ would be a definable bijection between a bounded and an unbounded definable set. By the Structure Theorem, it follows that there are $r_{0} \in(p, \infty), b \in R^{n}$ and $\lambda \in \Lambda^{n}$, such that

$$
\forall t \in\left(r_{0}, \infty\right), \varphi(t)=b+\lambda t
$$

We have thus found a normalized 1-cone $C=b+\lambda t$ contained in $K$ such that $\lim _{t \rightarrow \infty}(b+\lambda t)=\infty_{K}$. Let us fix the cone $C$. By Property (3) from the Introduction and the (IVT), it follows that $C=\left\{x \in K: x>_{K} \varphi\left(r_{0}\right)\right\}$.

Claim 2.5. Let $\langle K, *\rangle$ be a definable group with $\operatorname{dim}(K)=\operatorname{ldim}(K)=1$. Then $K$ is definably isomorphic to $\langle R,+\rangle$.

Proof. By [MS, Proposition 3.3], it suffices to show that $\left\langle K,<_{K}, *, 0_{K}\right\rangle$ is definably isomorphic to some definable ordered group $\langle R,<, \oplus\rangle$, where $<$ is the order of $\mathcal{R}$. It suffices to define a definable bijection $f:\left\langle K,<_{K}\right\rangle \longrightarrow\langle R,<\rangle$ that preserves the order. For $x \in C$, we let $f(x)=\varphi^{-1}(x)$. For $0_{K} \leq_{K} x<_{K} \varphi\left(r_{0}\right)$, we let $f(x)=\varphi^{-1}\left(\varphi\left(r_{0}\right) * x\right)-a$. For $x<_{K} 0_{K}$, we then let $f(x)=-\varphi^{-1}\left(-{ }_{*} x\right)$. We leave it to the reader to check that $f$ is as desired.

We are now ready to prove the main theorem of this paper. If $G$ is a definable group, then by $G^{0}$ we denote the definably connected component of the identity of $G$ with respect to the $t$-topology.

Theorem 2.6. Assume that $\mathcal{R}$ is semi-bounded and let $G=\langle G, \cdot\rangle$ be a definable group with $\lim (G)=m$. Then $G$ is a definable extension of a bounded definable group $B$ by $\left\langle R^{m},+\right\rangle$ :

$$
0 \longrightarrow\left\langle R^{m},+\right\rangle \longrightarrow G \longrightarrow B \longrightarrow 0 .
$$

Proof. We first remark that $G$ contains a definable radical $U$, that is, a maximal definably connected definable normal solvable subgroup. Indeed, if not, then we can construct a sequence of definable subgroups $0=B_{0}<\cdots<B_{l}<\cdots<G$, each definably connected, solvable and normal. But since for any two definably 
connected subgroups $K_{1} \leqslant K_{2}<G$, either $K_{1}=K_{2}$ or $\operatorname{dim}\left(K_{1}\right)<\operatorname{dim}\left(K_{2}\right)$, we derive an infinite sequence of subgroups of $G$ which have strictly increasing dimensions, a contradiction.

Now let $H=G / U$. By definable choice, we may assume that $H$ is a definable group, which is either finite or definably semi-simple, that is, it has no normal abelian definable subgroup of positive dimension. In either case, $H$ is bounded. Indeed, if $H$ is finite, then it is obviously bounded. If $H$ is definably semi-simple, then by [PPS], the quotient of $H^{0}$ by its finite center is a direct product $H_{1} \times \cdots \times$ $H_{k}$ of definable subgroups, such that for each $i \in\{1, \ldots, k\}$, there is a definable real closed field $\mathcal{R}_{i}$ and a definable isomorphism between $H_{i}$ and a semialgebraic subgroup of $\operatorname{GL}\left(n_{i}, R_{i}\right)$. Since $\mathcal{R}$ has no poles (Fact 2.1), each real closed field $\mathcal{R}_{i}$ is bounded, and so is each of $\operatorname{GL}\left(n_{i}, R_{i}\right)$ and $H_{i}$. Thus, $H^{0}$ and $H$ are bounded.

By [Ed2, Theorem 5.8], $U$ has a normal definable subgroup $V$ which is a direct product $V=W_{1} \times \cdots \times W_{s} \times V_{1} \times \cdots \times V_{k}$ such that the following hold:

(i) $U / V$ is a definably compact definable group, and thus it is bounded,

(ii) for each $j=1, \ldots, s$, there is a definable semi-bounded o-minimal expansion $\mathcal{J}_{j}$ of an ordered group such that $W_{j}$ is a direct product of copies of the additive group of $\mathcal{J}_{j}$,

(iii) for each $i=1, \ldots, k$, there is a definable o-minimal expansion $\mathcal{I}_{i}$ of a real closed field such that $V_{i}$ is definably isomorphic to an $\mathcal{I}_{i}$-definable group.

As above, since $\mathcal{R}$ has no poles, for each $i=1, \ldots, k$, the underlying definable real closed field of $\mathcal{I}_{i}$ is bounded, and so is each $V_{i}$. Thus, $V_{1} \times \cdots \times V_{k}$ is bounded. Moreover, let $S$ be the set of all $j \in\{1, \ldots, s\}$ such that the additive group of $\mathcal{J}_{j}$ is unbounded and set $W=\Pi\left\{W_{s}: s \in S\right\}$ and $E=\Pi\left\{W_{j}: j \in\{1, \ldots, s\} \backslash S\right\} \times$ $V_{1} \times \cdots \times V_{k}$. Then $E$ is bounded.

Note that $W$ is a definable normal subgroup of $G$. Indeed, first observe that $W$ is a definable subgroup of ( $U$ and thus of) $G$ of maximal linear dimension. Now let $g \in G$. We show that $g W g^{-1} \subseteq W$. Since $W$ is a direct product of copies of unbounded o-minimal ordered groups, so is $g W g^{-1}$. It suffices to show that if $L$ is one of those unbounded o-minimal subgroups of $\mathrm{gWg}^{-1}$, then $L$ is contained in $W$. Assume not. Then, since $W \cap L$ is a proper definable subgroup of $L$, it must be the trivial one. Hence, one can define an injective function $i: W \times L \longrightarrow W \cdot L$, $(w, l) \longmapsto w \cdot l$. By Fact 2.2(1,3), $\operatorname{ldim}(W \cdot L) \geq \operatorname{ldim}(W \times L)=\operatorname{ldim}(W)+\operatorname{ldim}(L)=$ $\lim (W)+1$, contradicting the maximality of $\operatorname{ldim}(W)$. This shows that $W$ is normal.

We have that:

(a) $G$ is a definable extension of $B=G / W$ by $W$,

(b) $B=G / W$ is a definable extension of $H=G / U$ by $U / W$, and

(c) $U / W$ is a definable extension of $U / V$ by $E$.

By (b) and (c), $B$ is in definable bijection with $H \times U / V \times E$, and thus by Fact $2.2(2,3), B$ is bounded.

By (a), $G$ is in definable bijection with $B \times W$. By Fact $2.2(2,3), \operatorname{ldim}(W)=$ $\lim (G)=m$. But $\operatorname{dim}(W)=\operatorname{ldim}(W)=m$ and thus, by Lemma 2.5, $W$ is definably isomorphic to $\left\langle R^{m},+\right\rangle$. Therefore, $G$ is a definable extension of the bounded definable group $B$ by $\left\langle R^{m},+\right\rangle$. 


\section{The Linear CASE}

We are going to use the following characterization of linear o-minimal expansions of ordered groups.

Fact $3.1([\mathrm{LP}]) . \mathcal{R}$ is linear if and only if there is an elementary extension of $\mathcal{R}$ which is a reduct of an ordered vector space $\mathcal{V}=\left\langle V,<,+, 0,\{d\}_{\lambda \in D}\right\rangle$ over an ordered division ring $D$.

For the rest of this section we assume that $\mathcal{R}$ is linear, and we fix $D$ as above.

Our goal below is to show that if a definable (in $\mathcal{R}$ ) group $K$ is bounded, then it is also definably compact. Since definable compactness is preserved under taking reducts, we may assume that $\mathcal{R}$ is $\mathcal{V}$.

A function $f: X \subseteq R^{n} \rightarrow R$ is called affine if there are $\lambda_{1}, \ldots, \lambda_{n} \in D$ and an element $a \in R$, such that for each $x=\left(x_{1}, \ldots, x_{n}\right) \in X$,

$$
f(x)=\lambda_{1} x_{1}+\cdots+\lambda_{n} x_{n}+a .
$$

Fact 3.2 ([LP]). Every definable function $f: X \subseteq R^{n} \longrightarrow R$ is piecewise affine; that is, there is a partition of $X=X_{1} \cup \cdots \cup X_{k}$ into finitely many definable sets, such that each restriction $f_{\uparrow X_{i}}$ is affine.

Lemma 3.3. Let $c<e<d$ in $R$. Then there is no definable family $h_{x}:[c, d) \longrightarrow$ $[x, d), x \in[e, d)$, of definable bijections.

Proof. Assume there is such a family. Let $H:[e, d) \times[c, d) \longrightarrow[c, d)$ with $H(x, t)=$ $h_{x}(t)$. We may assume that $H$ is affine. Indeed, since it is piecewise affine, there are $c^{\prime}<e^{\prime}<d$ in $R$ such that $H$ restricted to $\left[e^{\prime}, d\right) \times\left[c^{\prime}, d\right)$ is affine, and for each $x \in\left[e^{\prime}, d\right)$, the map $h_{x}$ restricted to $\left[c^{\prime}, d\right)$ is still a definable bijection.

So let $H(x, t)=\lambda t+\mu x+a$, for some fixed $\lambda, \mu \in D$ and $a \in R$. Without loss of generality, assume that $\forall x \in[e, d), h_{x}$ is increasing, that is, $h_{x}(d)=d$. We have:

$$
\begin{aligned}
& e=h_{e}(c)=\lambda c+\mu e+a \\
& d=h_{e}(d)=\lambda d+\mu e+a
\end{aligned}
$$

and, therefore, $\lambda(c-d)=e-d$.

Moreover, for every $x \in(e, d)$,

$$
\begin{gathered}
x=h_{x}(c)=\lambda c+\mu x+e \\
d=h_{x}(d)=\lambda d+\mu x+e,
\end{gathered}
$$

and, therefore, $\lambda(c-d)=x-d$. Thus $x=e$, a contradiction.

Claim 3.4. Let $K=\left\langle K, *, 0_{K}\right\rangle$ be a definable group. If $K$ is bounded, then it is definably compact.

Proof. We show that if $K \subseteq R^{n}$ is not definably compact, then it is unbounded. By [PeS], if $K$ is not definably compact, then it contains an one-dimensional definable subgroup which is not definably compact. It is thus sufficient to assume that $\operatorname{dim}(K)=1$.

We let $\varphi$ be as in the Introduction. We show that $\varphi((r, q))$ is unbounded. Assume, towards a contradiction, that it is bounded. Then $q \in R$. Let $s:[r, q) \longrightarrow$ $\left[\varphi^{-1}(\varphi(r) * \varphi(r)), q\right)$ be the map

$$
s(a)=\varphi^{-1}(\varphi(a) * \varphi(r)),
$$


and, for each $a \in[r, q)$, let $f_{a}:[r, q) \longrightarrow[s(a), q)$ be the map

$$
f_{a}(t)=\varphi^{-1}(\varphi(a) * \varphi(t)) .
$$

By Property (4) from the Introduction, each of the maps $s$ and $h_{a}, a \in[r, q)$, is easily seen to be a bijection. Thus, the family of maps $h_{x}:[r, q) \longrightarrow[x, q)$, $x \in[s(r), q)$, with

$$
h_{x}(t)=f_{s^{-1}(x)}(t)
$$

is a definable family of definable bijections that contradicts Lemma 3.3 for $c=r$, $e=s(r)$ and $d=q$.

Remark 3.5. The definition of a linear o-minimal structure $\mathcal{R}$ in $[\mathrm{LP}]$ is more general than the one we consider here. Namely, it includes the case where $\mathcal{R}$ does not expand an ordered group, but, rather, a "group interval". It is shown in [LP] that an $\mathcal{R}$ of the latter kind has an elementary extension which is a reduct of an interval of an ordered vector space $\mathcal{V}=\left\langle V,<,+, 0,\{d\}_{\lambda \in D}\right\rangle$ over an ordered division ring $D$. We observe that Theorem 1.5 trivially holds in that case. Indeed, any definable group in such an $\mathcal{R}$ is bounded, since its interpretation in $\mathcal{V}$ is.

\section{REFERENCES}

[vdD] L. van den Dries, TAme topology And o-minimal structures, Cambridge University Press, Cambridge, 1998.

[Ed1] M.Edmundo, Structure theorems for o-minimal expansions of groups, Ann. Pure Appl. Logic 102 (2000), 159-181.

[Ed2] M.Edmundo, Solvable groups definable in o-minimal structures, J. Pure Appl. Algebra 185 (2003), 103-145.

[EdOt] M. Edmundo and M. Otero, Definably compact abelian groups, J. of Math. Logic, 4 (2004), 163-180.

[ElSt] P. Eleftheriou and S. Starchenko, Groups definable in ordered vector spaces over ordered division rings, Journal of Symbolic Logic 72 (2007), 1108-1140.

[HPP] E. Hrushovski, Y. Peterzil and A. Pillay, Groups, measures, and the NIP, J. Amer. Math. Soc. 21 (2008), 563-596.

[LP] J. Loveys and Y. Peterzil, Linear o-minimal structures, Israel Journal of Mathematics 81 (1993), 1-30.

[MS] C. Miller and S. Starchenko, A growth dichotomy for o-minimal expansions of ordered groups, Trans. Amer. Math. Soc. 350 (1998), 3505-3521.

[Ot] M. Otero, A survey on groups definable in o-minimal structures, Preprint (2006).

[Pet1] Y. Peterzil, A structure theorem for semibounded sets in the reals, Journal of Symbolic Logic 57 (1992), 779-794.

[Pet2] Y. Peterzil, Returning to semi-bounded sets, Preprint (2007).

$[\mathrm{PeS}] \quad Y$. Peterzil and C. Steinhorn, Definable compactness and definable subgroups of ominimal groups, J. London Math. Soc. (2) 69 (1999), 769-786.

[PPS] Y.Peterzil, A.Pillay and S.Starchenko, Definably simple groups in o-minimal structures, Trans. Amer. Math. Soc. 352 (10)(2000), 4397-4419.

[Pi1] A. Pillay, On groups and fields definable in o-minimal structures, J. Pure Appl. Algebra 53 (1988), 239-255.

[Pi2] A. Pillay, Type definability, compact Lie groups, and o-minimality, J. of Math. Logic, 4 (2004), 147-162.

[Raz] V. Razenj, On one-dimensional groups over an o-minimal structure, Annals of Pure and Applied Logic 53 (1991), 269-277.

CMaf Universidade de Lisboa, Av. Prof. Gama Pinto 2, 1649-003 Lisboa, Portugal

E-mail address: edmundo@cii.fc.ul.pt

CMaf, Universidade de Lisboa, Av. Prof. Gama Pinto 2, 1649-003 Lisboa, Portugal E-mail address: pelefthe@ptmat.fc.ul.pt 